\title{
Un marco analítico para el estudio de las condiciones del empleo urbano en la frontera norte*
}

\author{
René Martín Zenteno Quintero**
}

\begin{abstract}
Durante los últimos años hemos sido testigos de un continuo proceso de reestructuración relacionado con transformaciones importantes en los mecanismos operatizos de los mercados de trabajo y en la regulación de las economías del sistema mundial capitalista. México no ha estado aislado de este proceso, particularmente por el interés que ha existido en atraer una mayor inversión de capital nacional e internacional. La apertura económica del país en términos de inversión extranjera productiva ha tenido sus mayores efectos locales en las ciudades fronterizas norteñas, donde más de 200000 (mayoritariamente jóvenes y mujeres) se emplean en trabajos asalariados y con protección social en empresas manufactureras exportadoras. La importancia de estudiar los mercados de trabajo y el empleo en la región fronteriza es doble. Por un lado, la naturaleza del trabajo se ha modificado como resultado del creciente proceso de incorporación de miles de trabajadores a empresas manufactureras exportadoras en la periferia del sistema mundial capitalista. Por otro, estas condiciones de empleo surgidas de una nueva división internacional del trabajo, coexisten con formas tradicionales de empleo distintivas de las economías de América Latina, como por ejemplo el trabajo por cuenta propia. El presente trabajo introduce un marco analítico para el estudio de las condiciones del empleo en los centros urbanos fronterizos. El marco fue diseñado para servir de guía conceptual en el desarrollo de una investigación empirica y comprensiva sobre la naturaleza del empleo urbano en la zona. A manera de conclusión, al final del trabajo se ilustra la utilidad de esta propuesta analítica para aprehender y entender impor. tantes diferencias en las características del trabajo, tales como el nivel educativo de los trabajadores, el tipo de ocupaciones desempeñadas y las remuneraciones percibidas.
\end{abstract}

\section{Introducción}

El objetivo de este artículo es introducir un marco analítico para el estudio de las condiciones del empleo urbano en los mercados de trabajo de la región fronteriza norte de México; este marco, con modificaciones mínimas, es también funcional en el análisis de cualquier estructura de empleo urbano de México o de otro país de América Lati-

* El presente artículo constituye una versión modificada y resumida de los capítulos 1 y 2 de la tesis doctoral del autor de la Universidad de Texas en Austin titulada On Depiciting Local Labor Markets in México: The Structure of Employment Conditions in Tijuana and Ciudad Juárez. El autor agradece los valiosos comentarios realizados al trabajo por Harley Browning, Marcela Cerrutti, Alfredo Hualde y por los evaluadores anónimos del mismo.

** Director del Departamento de Estudios de Población de El Colegio de la Frontera Norte. 
na. ${ }^{I}$ El marco fue diseñado para servir de guía conceptual en el desarrollo de una investigación empírica y comprensiva sobre la naturaleza del empleo urbano en la frontera norte. El fundamento del mismo estriba en su capacidad de organizar lógicamente varias dimensiones referidas a las condiciones laborales que diferentes perspectivas académicas han postulado para el estudio de las formas de empleo en América Latina. Las particularidades de la vida económica de la región fronteriza México-Estados Unidos y la disponibilidad de información de encuestas estructuradas de corte transversal tales como la Encuesta Nacional de Empleo Urbano (ENEU) fueron aspectos igualmente importantes en su diseño. ${ }^{2}$

El trabajo se compone de tres partes. En una primera sección se describe brevemente el contexto reciente de cambio económico y laboral, que hace cada día más complejo el estudio de la naturaleza del trabajo. La segunda sección introduce las diversas dimensiones que conforman el marco analítico para estudiar las condiciones del empleo urbano. Finalmente, y a manera de conclusión, se ilustra la utilidad de nuestra propuesta para capturar y entender importantes diferencias en las características de los trabajadores, tales como su nivel de educación, el tipo de ocupaciones que éstos desempeñan y, especialmente, las remuneraciones percibidas.

\footnotetext{
1 El término "mercado de trabajo" es utilizado en este trabajo en un sentido amplio. Si bien teórica y estrictamente este concepto se refiere al contexto institucional de intercambio del trabajo como mercancía, es decir, por un precio (salario) determinado, la altísima movilidad de mano obra que existe entre formas asalariadas y libres de ocupación en países como México permite a nuestro juicio una menor rigidez en el uso práctico del mismo. Además, el empleo tradicional en sí mismo representa una venta de trabajo. Braudel señala esto último con bastante elocuencia en su brillante visión histórica del capitalismo. En una de sus narraciones se lee: "Es perfectamente claro, sin embargo, que el mercado laboral -como una realidad y no como un concepto- no fue una creación de la era industrial. El mercado de trabajo fue el mercado en el cual el hombre se ofrecía a sí mismo, sin ninguno de sus medios tradicionales de producción... Todo lo que tenía para ofrecer eran su brazo o su mano, su «trabajo" en otras palabras. Y por supuesto su inteligencia o habilidades. El hombre que se contrataba o vendía a sí mismo de esta manera pasaba a través de la apertura estrecha del mercado fuera de la economía tradicional". (Braudel, 1992: 52).

2 La ENEU es una encuesta continua que provee información valiosa sobre empleo para los 34 centros urbanos más importantes del país. La utilidad de la información de esta encuesta ha sido mostrada previamente por varios trabajos (Oliveira, 1989; Browning y Zenteno, 1993; Cruz, 1993; Roberts, 1993, entre otros).
} 


\section{El contexto de cambio económico y laboral}

Hasta hace no mucho tiempo, un elevado crecimiento económico era ajeno a los altos niveles de productividad asociados con el mantenimiento de una parte significativa de la fuerza de trabajo laborando tiempo completo, en condiciones de empleo estables y sumamente protegidas en términos salariales y de beneficios laborales. El reconocimien to de este hecho, por parte tanto del capital como del trabajo, fue el principal ingrediente para la evolución de mercados de trabajo segmentados en la mayoría de los países capitalistas avanzados (Doeringer y Piore, 1971; Gordon, Edwards y Reich, 1982). Sin embargo, hoy en día todo parece indicar que el capital ya no requiere de este compromiso en los niveles y condiciones anteriores (por lo menos en algunas etapas del proceso productivo), a fin de tener éxito en un contexto de alta competitividad internacional y de nuevas lógicas económicas en la operación de los mercados productivos. Por lo contrario, previos arreglos institucionales entre empresas y trabajadores parecen presentar más bien serias barreras para las nuevas reglas de acumulación capitalista.

Durante las útimas dos décadas la economía capitalista mundial ha experimentado un periodo de recesión y de cambios estructurales significativos. La reestructuración de la economía capitalista mundial es el complejo resultado de factores como los mencionados por Lápple (1985: 43):

[...] erosión de las capacidades productivas y del empleo como consecuencia de la recesión; cambios de gran alcance en el abastecimiento de energía y materias primas; [...] estrategias del capital para una reorganización mundial de la producción y de la mercadotecnia conduciva a una relocalización internacional de recursos económicos; innovaciones tecnológicas radicales con implicaciones impredecibles para el empleo y las condiciones de vida de las poblaciones de diferentes países, y diversos tipos de luchas políticas y sociales y de intervención del Estado.

En un contexto mundial de fuerte competencia internacional y rápido cambio tecnológico, tanto los países capitalistas centrales como periféricos han sufrido las repercusiones de reestructuraciones económicas y sociales profundas cuyo fin es establecer los cambios institucionales necesarios que garanticen un crecimiento económico tan alto como en el pasado. La reorganización y relocalización mun- 
dial de la producción, y sus implicaciones sobre una creciente internacionalización del capital productivo, han sido mencionadas entre las características principales de la reestructuración económica en el mundo capitalista (Läpple, 1985). ${ }^{3}$ Según Walton (1985), el proceso de relocalización de capital y producción, de las economías măs avanzadas a los países periféricos en la forma de plantas de ensamble, representa una nueva y tercera división internacional del trabajo. ${ }^{4}$ Fróbel, Heinrichs y Kreye (1980: 45) han acuñado el término "nueva división internacional de trabajo" (NDIT) para

designar aquella tendencia que a) mina la bisección tradicional del mundo en unos pocos países industrializados por un lado, y una gran mayoría de países en desarrollo productores de materias primas por el otro y $b$ ) fuerza la subdivisión creciente de procesos de manufactura en un número de operaciones parciales en diferentes lugares industriales a través del mundo, donde la división del trabajo debe ser entendida como un proceso en movimiento y no como un resultado final.

La NDrT ha representado un problema de desempleo estructural en los países capitalistas măs avanzados y una industrialización en aumento en los menos desarrollados. Por un lado, los países centrales del sistema económico mundial han enfrentado serios problemas de desempleo masivo, subempleo, inestabilidad laboral y pobreza (Lăpple, 1985). Por otro lado, millones de trabajadores en los países periféricos se han incorporado a industrias manufactureras de exportación, muchos de los cuales pertenecen a grupos sociales tradicionalmente vulnerables en el mercado de trabajo como lo son losijóvenes y las mujeres (Barkin, 1985).

${ }^{3}$ Según Lápple (1985: 49), la reorganización de la producción en todo el mundo está caracterizada principalmente por dos tendencias: "primero, un crecimien to expansivo del capital, manifestado por la descentralización de la producción en una escala internacional, y cuyos principales objetivos son explotar la fuerza de trabajo barata en los países del Tercer Mundo y abrir nuevos mercados y esferas de acumulación; y segundo, una reestructuración intensiva de los sistemas productivos en los países capitalistas centrales, combinada con una lucha entre las corporaciones internacionales más poderosas por el control sobre sectores estratégicos de la economía mundial".

${ }^{4}$ La primera división internacional del trabajo se refiere a la postulada por Marx, esto es, la incorporación de regiones coloniales como nuevas abastecedoras de materias primas y consumidores para los países europeos industrializados. La segunda se refiere a la industrialización que tuvo lugar en la periferia a manos de una burguesía nacional, de empresas extranjeras o de ambas (Walton, 1985: 3-4). 
Es evidente que el proceso de reestructuración relacionado con prácticas flexibles de movilidad internacional de capital no se hubiera podido desenvolver sin serias transformaciones en los mecanismos operativos de los mercados de trabajo en los paises centrales y periféricos del sistema mundial capitalista. La reestructuración de los mercados de trabajo en Estados Unidos y Europa, por ejemplo, ha implicado la desregularización de diversos sectores económicos, la desorganización de importantes mercados laborales, la disminución del valor real de los salarios y un elevado desempleo abierto (Rosenberg, 1989). Los países de América Latina han experimentado transformaciones similares con el fin de atraer la inversión del capital nacional e internacional, así como para integrar totalmente sus economías al sistema internacional. Sin embargo, a diferencia de los países más avanzados, estos cambios han tomado lugar en sociedades abrumadas por viejos problemas de absorción laboral, condiciones de empleo pobres, bajos niveles salariales y, especialmente, por una gran desigualdad en la distribución del ingreso. Más aún, los países de la región intentan sobreponerse a consecuencias más severas de la crisis económica sobre los niveles de vida de su población.

Después de la segunda guerra mundial, varios años de rápido crecimiento económico e industrial fueron ciertamente críticos para la transformación de la fuerza de trabajo en América Latina. Bajo la estrategia de sustitución de importaciones, los efectos del proceso de industrialización fueron importantes para una movilización masiva de trabajadores de las áreas rurales a las urbanas, del autoempleo a relaciones asalariadas de producción, y del hogar a actividades económicas extradomésticas. No obstante estos avances, el crecimiento económico fue incapaz de producir empleos en los sectores modernos y más productivos al mismo ritmo que el aumento mostrado por la oferta de trabajo (Gregory, 1986; PREALC, 1976; Tokman, 1987). Así, los logros alcanzados en la creación de empleos productivos en la manufactura, el comercio y los servicios, fueron acompañados paralelamente por un crecimiento absoluto de ocupaciones en formas tradicionales de empleo en los principales centros urbanos de América Latina. Este patrón se pensó inicialmente como desfavorable para la calidad del empleo pero de naturaleza transitoria. El supuesto generalizado era que América Latina reproduciría finalmente la estructura de fuerza de trabajo mostrada por los países desarrollados (Gregory, 1986). Hoy en día sería muy dificil aceptar el carácter transitorio del trabajo informal, no asalariado, tradicional, irregu- 
lar, etc., como mucho del empleo de baja productivad ha sido denominado en diferentes estudios sobre el tema.

La incapacidad de las economías de América Latina para absorber un rápido crecimiento de la oferta de trabajo en relaciones de producción capitalistas ha sido exacerbada por la crisis y la reestructuración económica experimentada durante la pasada década. Asimismo, el panorama del empleo en la región se torna cada día más complejo en vista de la emergencia de nuevos patrones de trabajo irregular en los sectores modernos de la economía. Este proceso de precarización, resultado de la búsqueda de prácticas flexibles de contratación de mano de obra, ha implicado la transformación de muchos empleos productivos asalariados de tiempo completo, con amplias prestaciones laborales y de naturaleza estable, en diversas formas de trabajo temporal, de tiempo parcial, de pago a destajo o de nulos beneficios laborales extrasalariales.

No cabe duda de que los años ochenta serán recordados como uno de los periodos más críticos en la historia económica de América Latina (Canak, 1989). En el caso particular de México, estos años no fueron únicamente de crisis económica profunda, sino también de transformaciones significativas en los terrenos económicos, políticos y sociales. Después de casi cuarenta años de un crecimiento económico rápido y de mejoras notables en las condiciones de vida de la población mexicana, particularmente en las áreas urbanas, la primera mitad de la pasada década fue testigo de una importante reducción en el desarrollo social y económico de México, como consecuencia de circunstancias financieras críticas (Cordera y González, 1991; Lustig, 1992).

La crisis económica mexicana produjo altos niveles de desempleo abierto para el contex to de empleo urbano nacional (tasas superiores a $5 \%$ ), disminuciones sustanciales en el poder adquisitivo de los salarios, cambios en muchos contextos laborales regionales y sectoriales, un sector manufacturero nacional prácticamente estancado y, hasta 1987, altas tasas de inflación. En los años de severos ajustes recesivos de corto plazo (1982-1983), la producción mexicana se redujo $5 \%$, la tasa de desempleo abierto creció hasta $7 \%$, y los salarios industriales reales disminuyeron 25\% (PREALC, 1991).

En 1985, México pasó a formar parte del gatT (General Agreement on Tariffs and Trade), marcando con ello un parteaguas en la historia económica moderna del país. México se embarcó formalmente en un proyecto orientado a lograr una mayor integración de 
la economía nacional con el sistema económico mundial, dejando atrás muchas décadas de perseguir un modelo industrial proteccionista de sustitución de importaciones. Esto ha producido varios cambios en el país: la liberalización de su economía, la promoción de un modelo industrial orientado hacia el exterior, una mayor cabida a la inversión de capital foráneo, la reestructuración de las reglas de operación de muchos mercados de trabajo, una reducción seria del sector público vía la disminución de la burocracia o la venta de empresas estatales y, más tarde, la firma de un tratado de libre comercio con Estados Unidos y Canadá.

En otras palabras, México empezó a buscar fuertemente su integración al sistema económico mundial adoptando algunas de las políticas que la mayoría de los países capitalistas desarrollados habían iniciado como resultado de sus propias recesiones económicas. Sin embargo, los resultados han sido aparentemente más favorables para México dado que estas políticas han implicado un acceso indulgente a una fuerza de trabajo de muy bajo costo para el capital internacional. Así, después de otro periodo de ajuste económico como resultado de una nueva caída drástica de los precios del petróleo, México inició en 1986 un periodo de lenta recuperación económica. Entre 1986 y 1990 el PIB creció $2.5 \%$ anual, la inflación se redujo de $99 \%$ en 1982 , a $30 \%$ en 1989 y el desempleo abierto cayó de $6.6 \%$ en 1983, a 2.8\% al final de la pasada década (PREALc, 1991).

Según Lustig (1992: 116), la liberalización comercial, el relajamiento de restricciones para la inversión extranjera y la adaptación de la legislación mexicana a estándares internacionales, han sido los ingredientes esenciales de la política económica iniciada por el gobierno mexicano en 1985. Indudablemente, esta nueva política orientada a la reestructuración de la economía mexicana representa un viraje completo para la vida económica y social de la mayoría de las comunidades del país. Sin embargo, éste no es el caso de las localidades urbanas a lo largo de la frontera norte de México.

Durante la mayor parte del presente siglo, la frontera norte de México ha sido considerada como una región singular debido a que su población y economía han disfrutado de un régimen aduanal de zona libre y, más recientemente, de políticas especiales para el asentamiento de plantas con capital cien por ciento extranjero para la producción de ensamble. Estas regulaciones de excepcionalidad han estado históricamente orientadas a mejorar las condiciones de vida en una región con serios problemas para desarrollar una estructura pro- 
ductiva moderna y propia caracterizada además, por una gran dependencia económica respecto de Estados Unidos y por una débil integración con la economía nacional. ${ }^{5}$

En 1965, en momentos caracterizados por el alto crecimiento económico e industrial en el país y por una gran protección al capital nacional y rápida urbanización, el gobierno mexicano ofreció condiciones de excepción en las zonas y perímetros libres de la frontera norte con el fin de dar cabida a inversiones extranjeras. ${ }^{6}$ Las localidades de la frontera norte fueron, treinta años atrás, pioneras en la incorporación de México a los procesos de internacionalización del capital y de producción de ensamble. Aun cuando las maquiladoras (como estos establecimientos de capital extranjero son conocidos) y otras formas de empresas internacionales crecen actualmente más rápido en el interior del país, los efectos de la movilidad del capital y de la producción de países capitalistas avanzados son aún muy impresionantes en la región fronteriza. En 1990, 194651 empleos maquiladores se concentraban en tan sólo dos ciudades fronterizas (Tijuana y Ciudad Juárez), esto es, casi $40 \%$ del total nacional (véase gráfica 1). Si bien las condiciones de empleo han sido modificadas sustancialmente por el intenso crecimiento de la industria maquiladora en las comunidades fronterizas, especialmente durante la pasada década, aún existe una importante proporción de trabajadores laborando en formas de empleo tradicionales tales como el trabajo por cuenta propia, el trabajo familiar no remunerado y el servicio doméstico. Según datos de la ENEu, $21.4 \%$ y $24.7 \%$ de la población económicamente activa de Ciudad Juárez y Tijuana, los dos centros urbanos más importantes de la región, estaban empleados en estas condiciones en $1990 .^{7}$

5 El deseo de mejorar las condiciones de vida en la frontera fue relativamente más profundo en el pasado ante la fuerte necesidad de poblar la región. El poblamiento de la frontera se consideraba vital para frenar los afanes expansionistas de Estados Unidos.

${ }^{6}$ Con ello se inten tó reducir el alto desempleo en las localidades fronterizas producto del retorno de miles de trabajadores mexicanos que habían perdido sus trabajos con la terminación de los "programas de braceros".

7 Este apartado no incorpora en sú narración la crisis económica mexicana actual, que ha deteriorado seriamente las condiciones de vida de la población mexicana. Es importante únicamente mencionar que las reiteradas crisis económicas ponen de relieve la discusión sobre las condiciones de empleo en México y su frontera norte. En nuestra opinión, dos aspectos de las condiciones de empleo demandarán especial atención durante esta crisis en los ámbitos nacional y regional: el nivel de las tasas de desempleo abierto y la proporción de personas empleadas en actividades económicas informales. En el caso de la frontera norte será importante 


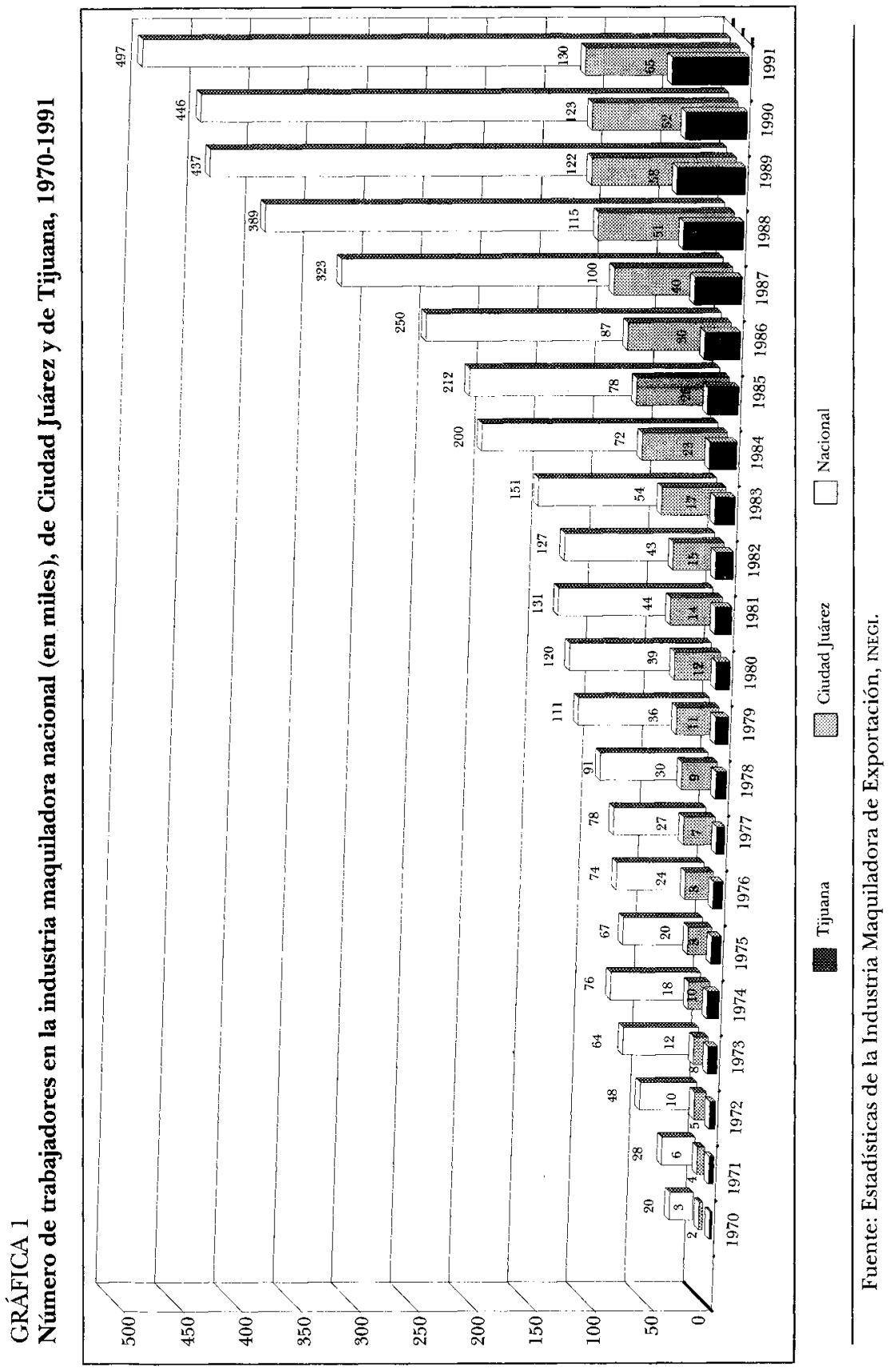


La importancia de estudiar los mercados de trabajo en la región fronteriza es por lo tanto doble. Por un lado, la naturaleza del trabajo se ha modificado como resultado del creciente proceso de incorporación de miles de trabajadores a empresas manufactureras exportadoras en la periferia del sistema mundial capitalista. ${ }^{8}$ Por otro lado, estas condiciones de empleo surgidas de una nueva división internacional del trabajo coexisten con formas tradicionales de empleo distintivas de las economías de América Latina. ${ }^{9}$

El estudio de los mercados de trabajo de la frontera norte representa una excelente oportunidad para avanzar en el conocimiento de la naturaleza de empleo urbano en México. El entendimiento de los mercados locales de trabajo en México es aún muy limitado, en parte debido al poco interés que los economistas mexicanos han mostrado por el tema en comparación con sus pares en otras partes del mundo. ${ }^{10}$ En ausencia de una economía laboral en México, la mayoría de los estudios sobre los mercados de trabajo y la estructura del empleo en el país han sido conducidos por sociólogos del trabajo, antropólogos sociales, demógrafos y algunos académicos con una sólida formación en economía política. Como es posible imaginarse, las perspectivas teóricas, metodologías, unidades de análisis, unidades de observación e información involucradas en sus estudios varían enormemente.

Los mercados de trabajo urbanos en la frontera norte de México han sido relativamente más estudiados (y probablemente también mejor estudiados) que en otras regiones y ciudades en México. Sin embargo, la mayoría de los estudios sobre empleo en esta parte del país ha emanado de análisis centrados en las empresas maquiladoras.

\footnotetext{
observar los niveles de trabajo asalariado pues la disminución del valor de la mano de obra en el contexto internacional muy probablemente atraiga un mayor número de inversiones y empleos relacionados con la producción de ensamble.

${ }^{8} \mathrm{La}$ internacionalización del capital ha ejercido una gran influencia en el aumento de los niveles de participación de la mujer en la actividad económica manufacturera. Estos empleos ofrecen ventajas para las mujeres (empleo de tiempo completo, seguridad social y otros beneficios), pero sin garantizar un grado importante de estabilidad en el trabajo y de oportunidades de movilidad ocupacional ascendente (Fernández-Kelly, 1983; Barajas y Rodríguez, 1989).

${ }_{9}^{9}$ Algunas de estas formas tradicionales de trabajo, como veremos al final de este trabajo, producen niveles de ingreso más altos que las formas modernas de empleo en la frontera. Esto debido en gran parte a que muchas de estas actividades económicas están orientadas hacia los consumidores estadunidenses.

${ }^{10}$ El trabajo más comprensivo de mercados de trabajo en México desde una perspectiva económica ortodoxa ha sido probablemente el desarrollado por el economista estadunidense Peter Gregory (1986).
} 
No cabe la menor duda de que las investigaciones sobre el empleo en la industria maquiladora son de suma relevancia debido al carácter internacional de la producción de ensamble, las características tan especiales de sus procesos productivos, y sus repercusiones en el aumento de la participación de las mujeres y los jóvenes en actividades económicas extradomésticas. Lamentablemente, el énfasis en el estudio de las maquiladoras no ha sido aparejado con esfuerzos similares para entender las características del empleo en otros sectores económicos. Más aún, no existe hoy en día un panorama comprensivo de los mercados de trabajo urbano en la frontera norte (Browning y Zenteno, 1993). El marco analítico que a continuación se presenta intenta modestamente contribuir a esta empresa.

\section{Un marco analítico para el estudio de la estructura de las condiciones del empleo urbano}

El problema de absorción laboral en América Latina ha sido conceptualizado desde muy diversas perspectivas. Informalidad, quizás, sea laescuela de pensamiento más difundida. Sin embargo, bajo el concepto de informalidad pueden ser encontradas diferentes y aparentemente antagónicas perspectivas (Roberts, 1990; Portes y Schauffler, 1993). En el caso particular de México, los estudios sobre empleo no han prestado mucha atención ni apreciado los análisis de la informalidad (esto debido principalmente a la multicitada imprecisión del concepto de informal). El problema del empleo en México ha sido abordado desde perspectivas más de índole desarrollista, marxista o económico-políticas, en las cuales se han enfatizado los éfectos que el desarrollo económico ha tenido en la transformación de la fuerza de trabajo, predominantemente en lo que respecta al proceso de proletarización del trabajo y a la evolución de las formas de empleo no asalariadas.

Otro grupo de estudios sobre el empleo en América Latina ha intentado conceptualizar cambios más recientes en las condiciones en que el trabajo productivo toma lugar, particularmente aquéllos orientados a establecer prácticas más flexibles de contratación y que tienden a manejar una mayor precariedad en el empleo de los sectores modernos de la economía (Rodgers, 1989; Marshall, 1987, 1990).

El marco analítico que se presenta en el diagrama 1 presenta y organiza lógicamente dimensiones propuestas por diversas conceptuali- 
ESTUDIOS DEMOGRÁFICOS Y URBANOS

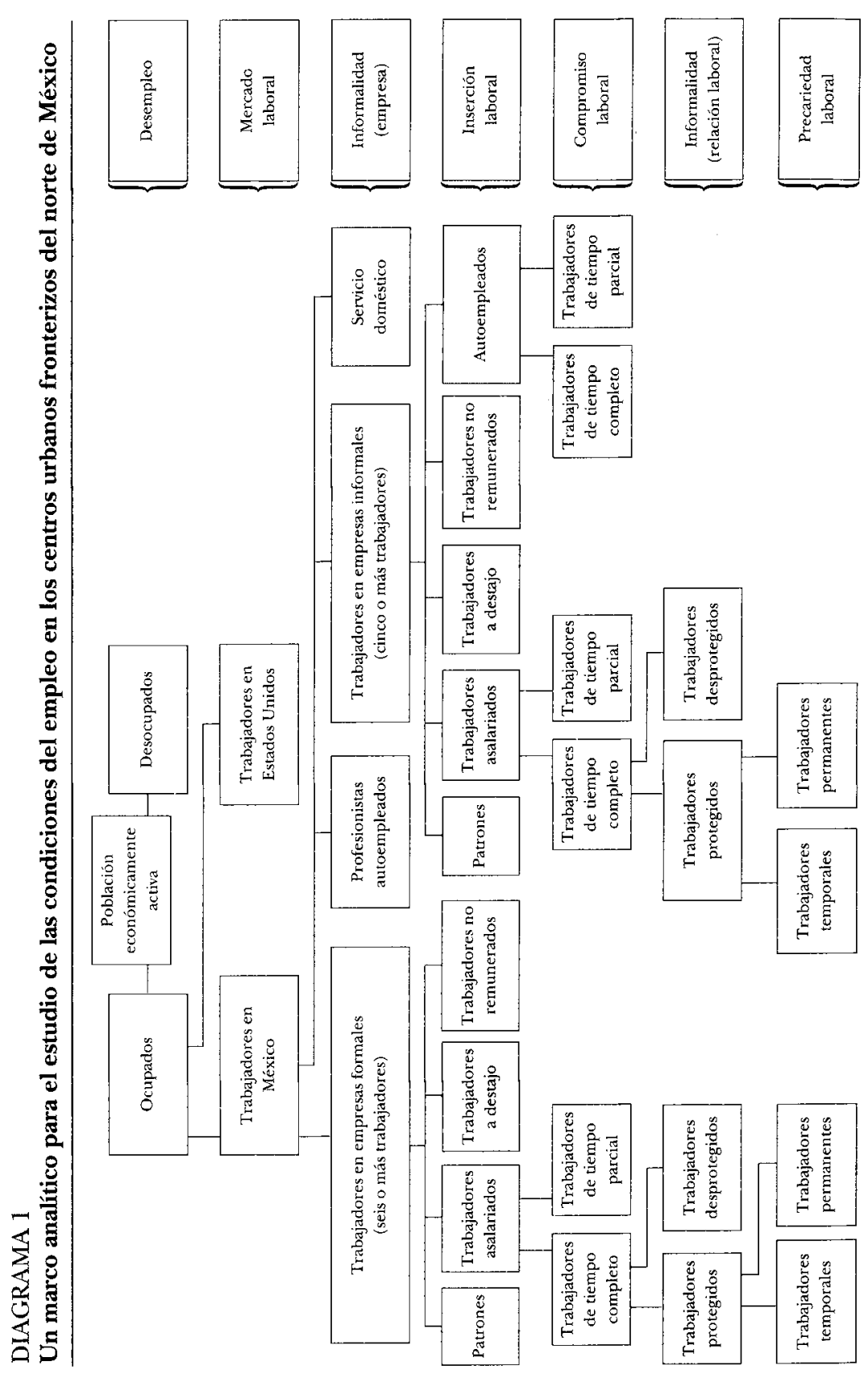


zaciones sobre la naturaleza del empleo en América Latina, mismas que están relacionadas con importantes aspectos de las condiciones del empleo. Las categorías que definen la estructura de condiciones de empleo descritas en este marco están organizadas en los siguientes siete niveles o dimensiones: 1) desempleo abierto; 2) mercado laboral; 3) informalidad laboral (la dimensión del tamaño de la empresa); 4) inserción laboral; 5) compromiso laboral; 6) informalidad laboral (la dimensión de la regulación de la relación laboral) y 7) precariedad laboral.

El marco fue diseñado como guía para desarrollar una investigación empírica y comprensiva sobre las condiciones del empleo en los mercados locales de trabajo urbano de la frontera norte, tomando en consideración el uso de información de corte transversal de encuestas estructuradas sobre empleo, como la ENEU. En su construcción también fue fundamental la decisión de no ceñirse a un solo enfoque teórico que pudiera limitar el análisis de la naturaleza del empleo a clasificaciones dicotómicas gruesas comúnmente utilizadas (formal e informal, asalariado y no asalariado, moderno y tradicional, regular e irregular, etc.), pues los enfoques duales presentan serias limitaciones para desarrollar caracterizaciones más integrales de las estructuras de oportunidades de empleo en América Latina. Por lo tanto, en este marco se privilegia la organización lógica de la riqueza conceptual y analítica que ha sido generada por las perspectivas más dominantes en el estudio de las formas de empleo en América Latina. ${ }^{11}$

Así pues, la estructura de condiciones de empleo que se deriva del marco propuesto está constituida por un arreglo de categorías relacionadas con los temas de informalidad, inserción de clase, compromiso laboral y precariedad. El marco intenta capturar diferentes niveles y formas de empleo que en su mayoría son aplicables a cualquier estructura urbana de América Latina. Sin embargo, fue también construido tomando en consideración las particularidades de la vida económica

11 Es importante mencionar que la decisión de no apegarse a un desarrollo conceptual particular está también relacionada con el hecho de que las características metodológicas de encuestas estructuradas de empleo no permiten examinar cabalmente las propociones conceptuales de enfoques como, por ejemplo, los de informalidad. Así, indagar sobre la discusión de informalidad presentada por Castells y Portes (1989), requeriría de información adicional proveniente de metodologías muy distintas a la que existe detrás de la ENEU. Éste sería el caso, por ejemplo, del intento de estudiar la funcionalidad que el sector informal mantiene con los sectores modernos de la economía, uno de los argumentos centrales en la discusión teórica de estos autores. 
de la frontera México-Ẹstados Unidos, y la posible disponibilidad de información de encuestas y censos. Con la excepción de las últimas categorías (trabajadores permanentes o temporales), todas las restantes pueden ser operacionalizadas con la información de la Encuesta $\mathrm{Na}$ cional de Empleo Urbano. ${ }^{12}$

\section{Desempleo abierto}

El universo de este marco lo constituye la población económicamente activa (PEA). En el nivel más general, la PEA se divide tradicionalmente en dos categorías amplias: ocupada y desocupada. La población económicamente activa desocupada se constituye por aquellas personas que buscan trabajo activa e ininterrumpidamente por un periodo determinado. ${ }^{13}$ El porcentaje de PEA desocupada es en la mayoría de las ocasiones mejor conocido como la tasa de desempleo abierto.

Los efectos que la recesión y la reestructuración económica han tenido sobre las condiciones de empleo de los países europeos y Estados Unidos, se han reflejado de manera significativa en el aumento del desempleo abierto. Sin embargo, los problemas de las condiciones de empleo en América Latina no habían sido, sino hasta muy recientemente, el desempleo abierto, el subempleo y la baja remuneración. ${ }^{14}$ Como bien ha señalado Mezzera (1987), el subempleo es el principal determinante de la pobreza de los hogares en América Latina. En un contexto de rápido crecimiento de la fuerza de trabajo, el

$12 \mathrm{Al}$ parecer ya es posible operacionalizar las últimas cuatro categorías con la información de la ENEU, puesto que recientemente se han incluido dos nuevas preguntas en el cuestionario sobre la naturaleza del contrato y la antigüedad en la ocupación principal.

${ }^{13}$ La ENEU define como población desocupada a aquellos individuos que buscan activamente trabajo durante las cuatro semanas previas a la encuesta.

14 Esta aseveración pierde cada día más fuerza en México. La crisis económica más reciente ha producido elevados niveles de desempleo abierto en todo el país. Al parecer, las oportunidades económicas en actividades de baja productividad (vía autoempleo o negocios familiares informales) empiezan a encontrar serios límites como mecanismo de ajuste de los mercados de trabajo, ante la creciente presión de millones de trabajadores que han perdido sus empleos y de cerca de un millón de nuevas personas en busca de trabajo que cada año se suman a la fuerza de trabajo en México. Esto significa que el desempleo abierto tomará cada vez mayor importancia como mecanismo de ajuste de los mercados de trabajo en el país y probablemente, adopte un carácter más estructural en la economía mexicana. 
desarrollo de actividades económicas de baja productividad ha sido sustituto de un sistema inadecuado de seguridad social en América Latina. Por lo tanto, el estudio de las condiciones de empleo en la región requiere de un gran entendimiento de la naturaleza del trabajo realizado por la población trabajadora, y eso es precisamente lo que este marco analítico intenta describir en las dimensiones restantes.

\section{Mercado laboral}

Las particularidades de la vida económica en la frontera México-Estados Unidos han producido un contexto en el que no necesariamente existe una total correspondencia entre el lugar de residencia y el lugar de trabajo. Este fenómeno puede ser ordinario en el contexto de muchas áreas metropolitanas alrededor del mundo. Sin embargo, transmigrar por motivos laborales (commuting) en la región fronteriza tiene algunas implicaciones singulares. Primero, la transmigración tiene un carácter binacional. Segundo, esta naturaleza binacional tiene un significado especial en el contex to de las grandes diferencias entre los mercados de trabajo de México y Estados Unidos. Tercero, las desigualdades entre las condiciones laborales de estos mercados de trabajo implican la existencia de barreras institucionales significativas para tener acceso a ellos, especialmente en el caso de los ciudadanos mexicanos que intentan trabajar en Estados Unidos. Ciertamente, podría señalarse que de existir una segmentación en los mercados de trabajo de esta región fronteriza, ésta estaría representada por el acceso de residentes mexicanos a trabajos en Estados Unidos.

La distinción de los mercados de trabajo es importante al intentar hacer un análisis comparativo adecuado de los mercados locales de trabajo en la frontera norte, especialmente si se desea contrastar a éstos con otros del interior del país, pues es necesario en este caso excluir del análisis a los residentes en las ciudades fronterizas que se emplean en Estados Unidos. Esta consideración es crítica cuando se trata de estudiar diferencias en los ingresos de los trabajadores. Si bien la economía y la población de las ciudades fronterizas mexicanas se benefician enormemente de los ingresos producidos por empleos en el país vecino, ${ }^{15}$ las políticas laborales para estos centros urbanos

15 Aproximadamente $20 \%$ del monto total de ingresos producto de la ocupación principal de la población trabajadora de la ciudad fronteriza de Tijuana, es 
deben basarse en el análisis de las condiciones de empleo prevalecientes en los mercados locales de trabajo. Sin embargo, si el interés de una investigación es comprender el empleo como una estructura de oportunidades para individuos y familias fronterizas, sería un error serio no considerar a los trabajadores en Estados Unidos.

Así pues, las disímiles características de los mercados de trabajo en uno y otro lado de la frontera, hacen necesaria la distinción de los residentes en la frontera mexicana que trabajan en México de aquéllos que laboran en Estados Unidos. Como veremos más adelante, el trabajo en Estados Unidos es muy importante para entender los altos niveles de ingreso de la población trabajadora en los centros urbanos fronterizos del norte de México.

\section{Informalidad laboral (el tamaño de la empresa)}

El tamaño de la empresa, la mayoría de las veces definido a partir del número de trabajadores, es una de las características de los mercados de trabajo más importantes que demarcan diferencias en condiciones de empleo. Por un lado, las empresas de mayor tamaño ofrecen salarios más altos y mejores oportunidades de movilidad como consecuencia de sus altos niveles de especialización y tecnología superior. En el caso de México, las empresas grandes tienen también más probabilidad de ofrecer pagos de seguridad social a sus trabajadores, son más propensas a ofrecer empleo estable de tiempo completo y otros beneficios sociales y económicos establecidos por las leyes del trabajo. Por otro lado, las empresas pequeñas tienden a descansar más en el trabajo familiar no remunerado y $(o)$ en el empleo asalariado sin seguridad social, con el objetivo de reducir costos en vista de su poca tecnología y capital. Los trabajadores de las empresas pequeñas se supone que experimentan las peores condiciones de empleo.

Los estudios sobre empleo en México han prestado muy poca atención a la dimensión de tamaño de la actividad económica más allá de comparar el trabajo por cuenta propia con el resto de la fuerza de trabajo. Sin embargo, estudios en otros países latinoamericanos han dispensado una mayor consideración a la única perspectiva que ha abordado el problema del empleo en un contexto de desarrollo

obtenido por tan sólo $7 \%$ de residentes que trabajan en Estados Unidos (Zenteno, 1992). 
de actividades económicas de muy pequeña escala y baja productividad: la informalidad.

En este particular marco analítico, el análisis de tamaño de la empresa se centra en el examen de los trabajadores que supuestamente laboran en las peores condiciones de empleo en América Latina, esto es, aquellos involucrados en empresas con menos de seis trabajadores. Esta operacionalización de tamaño de empresa, la cual incluye el trabajo por cuenta propia, evidentemente es muy cercana a la acuñada por la Oficina Internacional de Trabajo (OाT) para el estudio del sector informal. El Programa Regional sobre Empleo para América Latina y el Caribe (PREALc), oficina de la ort en América Latina, ha reconocido al sector informal como un sector económico compuesto por empresas de tamaño muy pequeño, que cuentan con muy poco capital y una tecnología muy básica. El sector informal ha sido la expresión principal de la sobreoferta de trabajo en los países menos desarrollados (Mezzera, 1987).

En el ámbito de la empresa, la informalidad es entendida en términos de la racionalidad inherente a los bajos niveles de productividad de las empresas o actividades económicas de pequeña escala. La falta de organización de las empresas pequeñas está relacionada con otras características económicas, como la ausencia de una separación clara entre capital y trabajo y las deficientes condiciones de empleo (PREALC, 1976, 1987; Tokman, 1977, 1987, 1991). Las empresas pequeñas tienen mayor probabilidad de apoyarse en fuerza de trabajo familiar sin remuneración y de escapar a regulaciones estatales concernientes a los pagos de seguridad social y al ofrecimien to de otros beneficios laborales a sus trabajadores. El empleo en el sector informal se supone es también de fácil acceso, muy competitivo, de baja o nula calificación y pobremente remunerado.

Las tres principales conclusiones de PREALC, después de muchos años de estudiar el sector informal en América Latina, han sido que: 1) éste no parece mostrar ninguna tendencia a su desaparición; 2) no es una fuente contracíclica a la riqueza y a la creación de empleo, y 3) no es homogéneo en términos de ingresos y capital (Roberts, 1990). ${ }^{16}$

${ }^{16} \mathrm{El}$ empleo en empresas pequeñas ha llegado a ser más significativo en América Latina. Según PREArc (1991), la principal característica del proceso de ajuste durante los años ochenta fue que una gran parte de los nuevos empleos generados correspondieron a empresas pequeñas y microempresas y $(o)$ a ocupaciones informales $y$, por lo tanto, a empleo de baja productividad. 
Al igual que los estudios de mercados de trabajo segmentados en Estados Unidos y en Europa, la estructura dual de empleo formal-informal es una clasificación dicotómica de empresas y empleo basada en la tecnología y calificación y características de los trabajadores. Desde una perspectiva económica, el problema de la informalidad está asociado a los bajos niveles de productivad que generan las empresas pequeñas (incluyendo el trabajo por cuenta propia) debido a su tecnología rudimentaria y escaso capital. Según Roberts (1990), el problema que enfrentan las empresas informales no es una excesiva regulación estatal, sino su naturaleza ineficiente. Desde una perspectiva sociológica el problema de la informalidad se relaciona con las características socioeconómicas y las pobres condiciones de empleo de los trabajadores insertos en actividades de esta naturaleza, esto es, los bajos niveles de calificación de su fuerza de trabajo, el intenso uso de trabajo familiar no remunerado, la falta de regulación estatal en términos de contratos laborales y cumplimiento de pagos de seguridad social y los supuestos bajos niveles de remuneración o ingreso.

La distinción formal-informal refleja dualismos comunes utilizados en el estudio de la naturaleza del empleo en países en vías de desarrollo. Nuestro interés en la discusión planteada por PREAIc en torno de la informalidad deriva de su utilidad para organizar lógicamente diferentes condiciones de empleo. La definición de informalidad de PREALC es importante en este marco analítico para diferenciar a los trabaijadores de empresas informales de aquéllos empleados en empresas más grandes y organizadas, más que para discutir sobre el llamado "sector informal". Las características de la información para la cual fue pensado este marco no permitiría esta tarea.

La dimensión de informalidad en el ámbito de la empresa, al igual que las restantes dimensiones en este marco analítico, hace referencia únicamente al empleo que toma lugar en México. La razón para ello es que la mayoría de las consideraciones conceptuales son más relevantes para el análisis de las características del empleo en países subdesarrollados donde existe una considerable sobreoferta de mano de obra. En este marco, el empleo en empresas informales se refiere a todos los trabajadores laborando en actividades económicas que involucran directamente a menos de seis trabajadores. Esta definición permite hacer comparaciones con estudios previos elaborados por PREALC para México y otros países de América Latina.

Como se puede apreciar en el diagrama 1, el trabajo profesional por cuenta propia y el trabajo de servicio doméstico son excluidos de es- 
ta definición de empleo informal: el primero, porque es muy dificil de suponer debido a que se trata de actividades de baja productividad. E1 segundo, porque este tipo de actividad no produce ningún servicio o producto para el mercado; además, bajo el concepto de empresa y en términos muy estrictos, los trabajadores del servicio doméstico no se emplean en una unidad productiva orientada al mercado.

\section{Inserción laboral}

Esta cuarta dimensión está relacionada con la estructura de inserción de clase, e intenta capturar la naturaleza diversa de las condiciones laborales dentro de las empresas formales e informales. La distinción de empleo formal e informal no es suficiente para capturar la multiplicidad de situaciones en lo que respecta a las condiciones de empleo. Varios autores ya han señalado que la simple dicotomía formal-informal es muy limitada en el contexto de la gran heterogeneidad de las economías urbanas de América Latina (Escobar, 1986; Pérez-Sáinz y Menjivar, 1991; Telles, 1988).

Así, la dimensión de inserción de clase está relacionada con el proceso de proletarización por medio del cual el trabajo independiente se transforma en trabajo asalariado (Browning y Singelman, 1978). El proceso de proletarización ha sido parcialmente desarrollado en sociedades capitalistas como la mexicana, donde la creación de empleos asalariados por los sectores económicos más modernos y productivos no ha sido suficiente para absorber un crecimiento más rápido de la oferta de trabajo.

En el caso de México, una vasta mayoría de los estudios sobre la naturaleza del empleo se ha basado en análisis empíricos de la situación de clase. Esto ha sido posible debido a que la variable "posición en la ocupación" se ha incluido sistemáticamente en censos y encuestas. Al dividir a los trabajadores en patrones, asalariados, autoempleados y trabajadores no remunerados, diversas investigaciones han tratado de medir e interpretar en qué grado la oferta de trabajo ha sido integrada a relaciones capitalistas de producción (proletarización), al igual que entender la sobrevivencia de formas de empleo no asalariadas, como el trabajo familiar no remunerado y el trabajo por cuenta propia. ${ }^{17}$

17 El énfasis en la variable posición en la ocupación está también asociado a otros tres fenómenos de empleo relacionados entre sí: la baja capacidad del sector 
El lugar central de la dimensión de inserción de clase es reflejado por los trabajos recientes de García (1988) y Rendón y Salas (1987, 1992), quienes han enfocado su atención a la deficiente penetración del capitalismo en México y al reconocimiento de formas de empleo nocapitalistas como una característica intrínseca de la estructura económica del país. Según Rendón y Salas (1992), la persistencia de relaciones no capitalistas de producción y de bajos niveles de ingreso entre la población trabajadora en México, en comparación con los países más desarrollados, representa el problema estructural más significativo del empleo en México.

Con ello, la dimensión de inserción de clase es importante no sólo para entender las diferentes clases de trabajadores como patrones (burguesía) y trabajadores asalariados (proletariado), sino también para comparar a estos trabajadores con aquéllos empleados en formas no estrictamente capitalistas. La dimensión inserción de clase es también relevante para comprender una de las formas precarias de empleo que ganan cada día mayor espacio como parte de la reestructuración de los mercados de trabajo: el trabajo a destajo.

\section{Compromiso laboral}

La dimensión de compromiso laboral está enfocada a diferenciar únicamente a los trabajadores que laboran de tiempo completo de aquéllos que lo hacen de forma parcial. Mientras que la dimensión de inserción de clase se refiere a la condición del trabajador en términos de la existencia o no de una relación patrón-empleado, la dimensión de compromiso laboral alude a la condición del trabajador en términos del grado de compromiso que existe entre el capital y el trabajo, en el caso de los trabajadores asalariados, y también a la naturaleza del compromiso de algunos trabajadores por cuenta propia. El término de "trabajo sin compromiso" (uncommited labor) fue introducido por Roberts (1989) para referirse a las diferentes situaciones de empleo en las que, ya sea por factores de demanda u oferta, empresas e individuos no estaban totalmente comprometidos a ofrecer o buscar trabajo de manera regular (asalariada, estable, protegida y de tiempo completo).

manufacturero para generar nuevas y majores ocupaciones; la terciarización del empleo y el incremento de la participación de la mujer en la actividad económica. 
La distinción aquí planteada tiene un mayor significado cuando se aplica al trabajo asalariado y al autoempleo. Por un lado, el proceso de flexibilización alrededor del mundo ha implicado un mayor uso de trabajo asalariado de tiempo parcial y temporal con el fin de evitar algunas obligaciones laborales de seguridad social. Por otro lado, si bien la falta de compromiso es determinada en la mayoría de las situaciones por factores de demanda, también es cierto que algunos miembros del hogar no muestran mucha preferencia por trabajar tiempo completo, debido principalmente a obligaciones de carácter doméstico o educativo. El empleo irregular, muchas veces de tiempo parcial y temporal, puede ser preferido por los miembros del hogar llamados "productores secundarios de ingresos" (Telles, 1988). Éste es el caso muchas veces del trabajo por cuenta propia de tiempo parcial, el cual está relacionado con la falta de oportunidades en formas regulares de empleo, así como con la facilidad que este tipo de empleo ofrece a las condiciones de ciertos individuos.

Finalmente, la diferenciación del trabajo de tiempo completo y parcial entre los trabajadores asalariados es también importante porque las empresas no pueden eludir, bajo ninguna circunstancia marcada por la ley del trabajo, los pagos de seguridad social a los trabajadores asalariados de tiempo completo. La protección laboral derivada de la seguridad social es una importante condición en el ámbito del trabajo, que define la informalidad laboral en el nivel de las relaciones laborales.

\section{Informalidad laboral (la regulación de la relación laboral)}

Una conceptualización diferente de la informalidad proviene de una perspectiva económico-política que centra su atención en la regulación estatal como determinante externo del sector o de la economía informal (Roberts, 1990). Aquí, la informalidad no es ni una condición individual ni una condición de empresa, sino un proceso de relación de producción y generación de ingreso, cuya característica central es su falta de regulación por instituciones sociales (Castells y Portes, 1989). Así, informal es aquello que escapa a regulaciones tales como leyes del trabajo, obligaciones fiscales, etc. (Roberts, 1991). Las actividades informales son también caracterizadas por una separación vaga entre capital y trabajo, por la inexistencia de una relación contractual entre ambos y por una fuerza de trabajo cuyas condiciones de trabajo y pago no son registradas por la ley (Portes y Johns, 1989). 
Existe un fuerte debate entre quienes manejan esta perspectiva de informalidad y aquéllos de PREALC. ${ }^{18}$ Sin embargo, no son objetivos de este trabajo ni describir este debate (que puede ser mejor apreciado en los trabajos mencionados en la nota anterior), ni definir posición alguna con respecto a estas perspectivas. Ambas escuelas de pensamiento sobre la informalidad parten de muy diferentes antecedentes académicos y preguntas de investigación, lo que permite que éstas contribuyan a llamar la atención sobre diferentes problemas relacionados con la operación de la economía, los mercados de trabajo y la estructura de empleo. Además, como se señaló anteriormente, el uso de información transversal de encuestas estructuradas de empleo serían insuficientes para comprender y analizar cabalmente cualquiera de estos enfoques.

Es importante mencionar que la razón para introducir previamente en el marco analítico la noción de tamaño de empresa tiene que ver con el hecho de que este importante aspecto estructural del mercado de trabajo determina en gran medida la capacidad de eludir las regulaciones laborales marcadas por la ley, pues a mayor tamaño de la empresa menor es la probabilidad de eludir las regulaciones del Estado.

Informalidad en el ámbito de las relaciones laborales se refiere pues a la protección de los trabajadores asalariados de tiempo completo. Por protección entendiendo la condición de recibir los beneficios laborales establecidos por la ley del trabajo, tales como: servicios de salud, aguinaldo, reparto de utilidades y vacaciones pagadas. Esta distinción se restringe únicamente a los trabajadores asalariados de tiempo completo, pues los códigos laborales no son ambiguos con respecto a la obligación de las empresas de ofrecer el tipo de beneficios mencionados (la ley del trabajo es incierta en el caso de trabajadores a destajo y aquéllos de tiempo completo).

La dimensión de informalidad aquí utilizada permite describir no únicamente el carácter de las condiciones de empleo no reguladas por el Estado, sino también la posible naturaleza inestable del empleo. Al definir como trabajadores asalariados protegidos de tiempo completo a aquellos que reciben por parte de las empresas seguridad social (IMSs, ISsSTE, etc.), vacaciones pagadas y aguinaldo, se logra a la

18 Pueden verse, por ejemplo, las discusiones entre Klein y Tokman (1988) y Portes (1989) en la revista mexicana Estudios Sociológicos. Un debate más reciente ha sido planteado por un trabajo de Portes y Schauffler (1993). 
vez una aproximación a la idea de estabilidad pues muy difícilmente estas prestaciones se obtienen en empleos de muy corto plazo. 19

\section{Precariedad laboral}

Una de las condiciones laborales más cambiantes alrededor del mundo tiene que ver con la transformación de ciertas ocupaciones de carácter permanente y estable en situaciones de tipo temporal e inestable. En este marco analítico la dimensión de precariedad laboral intenta comprender el deterioro que resulta en el aumento del empleo de tiempo completo, asalariado, protegido pero de naturaleza inestable. Esto es importante para poder entender la situación del empleo generado por la industria maquiladora de exportación en la frontera norte.

Empresas en los sectores económicos modernos alrededor del mundo han implantado nuevas reglas en la operación de los mercados de trabajo, orientadas a emplear trabajadores con bases más flexibles de contratación y despido que en el pasado. Desde el punto de vista de la demanda, este proceso de flexibilización existe como una estrategia de adaptación a la cada vez mayor competencia internacional y a los cambios impredecibles de los mercados productivos y de los ciclos económicos. El proceso de flexibilización laboral alrededor del mundo se ha materializado en la estructura del empleo de muy diversas maneras: un mayor uso del trabajo subcontratado, incremento en el empleo asalariado sin seguridad social, fragmentación de ocupaciones de tiempo completo enijornadas de tiempo parcial, disminución del número absoluto de empleos con contratos permanentes, etc.

Bajo cualquiera de las conceptualizaciones anteriormente mencionadas sobre el problema del empleo en América Latina, el trabajo en las maquiladoras sería conceptualizado positivamente en lo que

${ }^{19}$ Este supuesto es claramente más débil día con día. El concepto de "protección laboral" o "trabajo protegido", operacionalizado por medio de la cualidad del empleo de contar con todas las prestaciones marcadas por los códigos laborales, se aleja cada vez más de la noción de estabilidad laboral que se le solía adjudicar en el pasado. Actualmente, los bajos niveles salariales en México permiten que muchas empresas ofrezcan sin grandes problemas cientos de miles de empleos "protegidos" de naturaleza temporal o inestable. Esto es particularmente cierto en el caso las empresas manufactureras extranjeras. 
respecta a las condiciones de empleo: asalariado de tiempo completo y doblemente formal (tanto los criterios de tamaño de empresa como por los de protección laboral). Los empleos generados por empresas transnacionales en México y que se derivan de procesos de subcontratación internacional, no pueden, por su naturaleza, escapar a las regulaciones estatales. Además, el bajo costo de la mano de obra mexicana en el contexto internacional no representa ninguna barrera al pago de trabajo asalariado y al otorgamiento de beneficios laborales. Sin embargo, el trabajo en las maquiladoras de la frontera norte debe ser más discutido con el fin de entender algunos aspectos de precariedad. Esto por dos razones. Primero, porque estas empresas ofrecen empleo a una fuerza de trabajo considerada tradicionalmente como secundaria y muy vulnerable, es decir, jóvenes, mujeres y migrantes recién llegados a las ciudades fronterizas. Las maquiladoras prefieren contratar individuos con estas características sociodemográficas por varias razones, destacando entre las más importantes el hecho de que estos grupos sociales son menos tendientes a generar conflictos y a demandar un alto grado de estabilidad en el empleo. Segundo, relacionada estrechamente con la anterior, los empleos producidos por las maquiladoras ofrecen muy poca estabilidad en el trabajo. La mayoría de sus contratos son de naturaleza temporal y las posibilidades de promoción dentro de estas empresas son muy limitadas. Así pues, esta última dimensión intenta explicar el grado de estabilidad que los trabajadores asalariados de tiempo completo y protegidos tienen en su ocupación principal, tanto en las empresas formales como en la industria maquiladora y las empresas informales.

A manera de conclusión: una ilustración breve de los alcances del marco analítico para el estudio de los mercados locales de trabajo

En las páginas precedentes se ha planteado un marco análitico para el estudio de las condiciones del empleo urbano en las ciudades fronterizas del norte de México. El trabajo ha mostrado que es posible examinar con mayor detalle la estructura de las condiciones de empleo, al combinar en un orden lógico formas de empleo propuestas por diversas conceptualizaciones del problema de absorción laboral en América Latina. Este procedimiento permite avanzar analíticamente 
en la descripción de la cada vez más compleja situación laboral en América Latina y, a la vez, evitar con ello clasificaciones dicotómicas comunes utilizadas para su estudio.

El marco analítico aquí presentado se puede sintetizar en una sola variable. El cuadro 1 presenta las categorías de la variable "estructura de condiciones de empleo", y la misma representa el máximo nivel de desagregación posible de este marco. Dependiendo del interés por destacar algunas dimensiones más que otras y de los alcances muestrales de la fuente de información que se utilice, es posible reagrupar estas categorías con el fin de trabajar en distintos niveles de desagregación.

CUADRO 1

Estructura de condiciones de empleo

1 Población desocupada

2 Patrones de empresas formales

3 Profesionistas por cuenta propia

4 Trabajadores asalariados permanentes de tiempo completo y protegidos en empresas formales

5 Trabajadores asalariados temporales de tiempo completo y protegidos en empresas formales

6 Trabajadores asalariados de tiempo completo y desprotegidos en empresas formales

7 Trabajadores asalariados de tiempo parcial en empresas formales

8 Trabajadores a destajo en empresas formales

9 Trabajadores no remunerados en empresas formales

10 Patrones de empresas informales

11 Trabajadores asalariados permanentes de tiempo completo y protegidos en empresas informales

12 Trabajadores asalariados temporales de tiempo completo y protegidos en empresas informales

13 Trabajadores asalariados de tiempo completo y desprotegidos en empresas informales

14 Trabajadores asalariados de tiempo parcial en empresas informales

15 Trabajadores a destajo en empresas informales

16 Trabajadores no remunerados en empresas informales

17 Trabajadores informales por cuenta propia de tiempo completo

18 Trabajadores informales por cuenta propia de tiempo parcial

19 Trabajadores en servicio doméstico

20 Trabajadores en el mercado laboral de Estados Unidos 


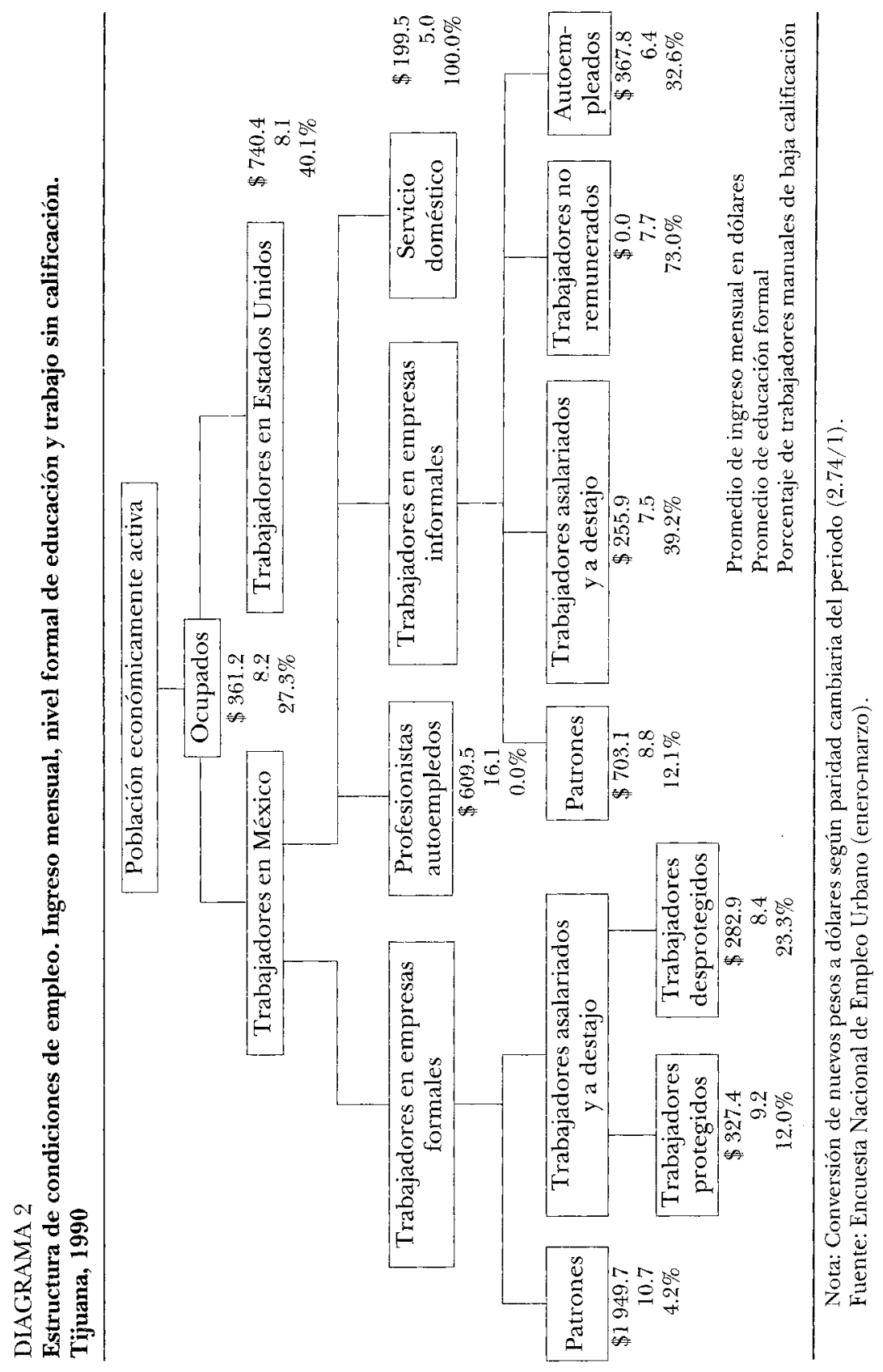


El esfuerzo por diseñar este marco análitico sería infructuoso de no poder demostrar su capacidad de diferenciar características relevantes del empleo, particularmente en lo que a producción de ingresos se refiere. Esto último es importante de señalar pues cada vez son más frecuentes las discusiones conceptuales acerca del problema del empleo en México (e investigaciones empíricas que se derivan de éstas) y que ponen muy poca atención a la relevancia que deben tener para el estudio de las grandes desigualdades en el ingreso). Es importante recordar que las discusiones teóricas sobre los mercados de trabajo y la estructura del empleo en los países capitalistas avanzados y en América Latina en los años sesenta, tuvieron como objetivo principal formular explicaciones para la gran heterogenidad salarial existente entre la población trabajadora a pesar de las tasas elevadas de crecimiento económico experimentadas en la posguerra. Esto es, el desarrollo de conceptualizaciones que permitieran explicar la inequidad salarial no asociada a los factores del capital humano. La estructura de condiciones de empleo aquí presentada es analíticamente relevante en la medida en que esté asociada significativamente a la producción diferencial de ingresos de los individuos (y sus hogares), una vez tomadas en cuenta variables de capital humano (educación, experiencia laboral, etc.) y otras características estructurales del mercado laboral (ocupación, rama de actividad, horas trabajadas, etcétera).

Obviamente que este espacio es insuficiente para intentar demostrar este complejo y laborioso análisis. Sin embargo, es posible mostrar la relación directa que guarda la estructura de condiciones de empleo derivada de este marco, con las diferencias que se presentan en la producción de ingresos. El diagrama 2 muestra el ingreso promedio mensual de los trabajadores de la ciudad fronteriza de Tijuana en 1990, para cada una de las condiciones de empleo que se derivan de una forma abreviada del marco analítico general. ${ }^{20}$ Además, este diagrama incluye información sobre el promedio de educación formal y el porcentage de trabajadores en ocupaciones manuales de baja calificación en cada una de estas once condiciones de empleo. Los resultados son por demás interesantes.

El promedio de ingreso mensual de la población trabajadora residente en Tijuana era equivalente a 361 dólares durante el trimestre

20 El marco analítico abreviado es necesario ya que el tamaño de muestra de la ENEU para Tijuana no es suficientemente grande para proporcionar confiablemente esta información con las 19 categorías terminales que se derivan del marco general. 
enero-marzo de 1990. A este ingreso mensual le correspondía una instrucción formal promedio de aproximadamente segundo año de educación secundaria (8.2 años), y $27 \%$ de los trabajadores desempeñaba ocupaciones manuales de baja calificación.

Las grandes diferencias entre los mercados de trabajo de México y Estados Unidos son evidentes. Con una educación formal similar (8.1 años) y con un mucho mayor desempeño en ocupaciones manuales de baja calificación (40\%), los residentes de Tijuana que laboran en Estados Unidos recibían una remuneración promedio cien por ciento superior a la obtenida por el total de la población trabajadora. Un contraste más evidente de esta situación surge al observar cómo los trabajadores profesionistas por cuenta propia, con una educación formal promedio de 16 años, ganaban 130 dólares menos que los trabajadores en Estados Unidos.

De las condiciones de empleo con remuneración, el servicio doméstico posee el nivel de ingresos más bajo con alrededor de 200 dólares mensuales. En este caso existe una total correspondencia entre el ingreso, la educación y la calificación del trabajo desarrollado. El resto de las condiciones de empleo muestran la heterogeneidad laboral que existe dentro de las empresas formales e informales.

La necesidad y utilidad de desagregar las condiciones de empleo de las empresas informales es claramente demostrada al observar la heterogeneidad en ingresos, educación y ocupación. Los patrones constituyen una condición de empleo previlegiada dentro de las empresas informales. La educación formal de los patrones es de tan sólo un año superior a la de los empleados remunerados en empresas informales. Sin embargo, el ingreso promedio de los primeros es $174 \%$ más alto que el de los segundos, hecho que está estrechamente vinculado al bajo nivel de participación de los patrones en ocupaciones manuales de baja calificación.

Los empleados remunerados (asalariados y a destajo), los trabajadores no remunerados y los trabajadores por cuenta propia en actividades económicas informales muestran niveles inferiores de educación formal a los de la población trabajadora total, así como una mayor tendencia a laborar en ocupaciones manuales de baja calificación. Los datos permiten apreciar dos resultados interesantes. Primero, el nivel tan alto de ocupación de los trabajadores no remunerados en actividades manuales de baja calificación, en comparación con el resto de los trabajadores empleados en empresas informales, a pesar de contar con una educación promedio similar a los trabajadores asalariados y a destajo, y superior a la de los trabajadores por cuenta pro- 
pia. Esto advierte claramente de la existencia de un claro subempleo entre los trabajadores no remunerados, muchos de los cuales se encuentran en una situación de sacrificio (o explotación) en aras del bienestar de las empresas familiares en que laboran.

Segundo, el promedio tan elevado de ingresos de los trabajadores por cuenta propia en actividades informales. Los trabajadores por cuenta propia de Tijuana tienen un ingreso promedio superior al de los trabajadores protegidos y desprotegidos de empresas formales, a pesar de su menor educación (sólo primaria completa) y su mayor probabilidad de desempeñar actividades de baja calificación (33\%). Dos factores están relacionados con este resultado. Primero, que el ingreso no toma en cuenta el valor monetario de las prestaciones que reciben los trabajadores protegidos en empresas formales, por lo que la diferencia entre estos trabajadores y los autoempleados está sobreestimada. Segundo, que las actividades informales en la frontera norte son capaces de producir ingresos elevados dada su orientación al turismo estadunidense, lo cual representa tener acceso a ingresos en dólares, oportunidad que no encuentran los trabajadores formales.

Los trabajadores protegidos y desprotegidos de empresas formales constituyen clases de trabajadores claramente distintas. La protección laboral de los trabajadores en este tipo de empresas representa una condición de empleo altamente correlacionada con una mayor educación formal, una menor probabilidad de desempeñar ocupaciones manuales de baja calificación, y mejores condiciones de remuneración económica. Finalmente la información muestra notoriamente las condiciones de empleo privilegiadas de los patrones de empresas formales.

Así pues, este trabajo de investigación ha presentado un marco analítico cuyo objetivo es servir de guía para el estudio de las condiciones del empleo urbano en los mercados de trabajo de la frontera norte. Su aplicabilidad obviamente se extiende más allá de la frontera norte y sus alcances están aún por ser determinados en investigación empíricas futuras. Por ahora se ha podido simplemente ilustrar que la estructura de condiciones de empleo está estrechamente relacionada con otras características relevantes de los mercados de trabajo. 


\section{Bibliografía}

Barajas, Rocío y Carmen Rodríguez (1989), "Mujer y trabajo en la industria maquiladora de exportación", Documentos de Trabajo Fundación Friedrich Ebert, núm. 22, México, Fundación Friedrich Ebert.

Barkin, David (1985), "Global Proletarization”, en Steven Sanders (ed.), The Americans in the New International Division of Labor, Londres, Holmes and Meir, pp. 26-45.

Braudel, Fernand (1992), The Wheels of Commerce. Civilization $\mathcal{E}$ Capitalism 15th-18th Century, Los Angeles, University of California Press, vol. 2.

Browning, Harley y René Zenteno (1993), "The Diverse Nature of the Mexican Northern Border: The Case of Urban Employment", Frontera Norte, vol. 5, núm. 9, pp. 11-31.

y Joachim Singelmann (1978), "The Transformation of the U.S. Labor Force: The Interaction of Industry and Occupation", Politics $\mathcal{E}^{2} S o-$ ciety, vol. 8, núms. 3-4, pp. 481-509.

Canak, William L. (1989), "Debt, Austerity, and Latin America in the New International Division of Labor", en William L. Canak (ed.), Lost Promises. Debt, Austerity, and Development in Latin America, Boulder (Colorado), Westview Press, pp. 9-27.

Castells, Manuel y Alejandro Portes (1989), "World Underneath: The Origins, Dynamic, and Effects of the Informal Economy", en Alejandro Portes et al. (eds.), The Informal Economy: Studies in Advanced and Less Developed Countires, Baltimore, Johns Hopkins University Press, pp. 11-37.

Cordera Campos, Rolando y Enrique González Tiburcio (1991),"Crisis and Transition in the Mexican Economy", en Mercedes González de la Rocha y Agustín Escobar Latapí (eds.), Social Responses to Mexico's Economic Crisis of the 1980's, San Diego, Center for U.S.-Mexican Studies, University of California, pp. 19-56.

Cruz, Rodolfo (1993), "Algunos factores asociados a la participación femenina en los mercados de trabajo: ciudades de la frontera y áreas metropolitanas de México", Frontera Norte, vol. 5, núm. 9, pp. 97-116.

Doeringer, P. y Michael Piore (1971), Internal Labor Markets and Manpower Analysis, Lexington (Mass.), Heath Lexington Books.

Escobar Latapí, Agustín (1986), Con el sudor de tu frente. Mercados de trabajo y clase obrera en México, Guadalajara, EI Colegio de Jalisco.

Fernández-Kelly, María Patricia (1983), "Mexican Border Industrialization, Female Labor Force Participation, and Migration", en June Nash y Maria Patricia Fernández-Kelly (eds.), Women, Men, and the International Division of Labor, Albany (Nueva York), State University of New York Press, pp. 205-223.

Fröbel Loker, Jurgen Heinrichs y Otto Kreye (1980), "The New International Division of Labor: Structural Unemployment", en Industrialized Countries and Industralization in Developing Countries, Cambridge, Cambridge University Press. 
García, Brígida (1988), Desarrollo económico y absorción de fuerza de trabajo en México: 1950-1980, México, El Colegio de México.

Gordon, David, Richard Edwards y Michael S. Reich (1982), Segmented Work, Divided Workers: The Historical Transformation of Labor in The United States, Cambridge, Cambridge University Press.

Gregory, Peter (1986), The Myth of Market Failure. Employment and the Labor Market in Mexico, Baltimore, Johns Hopkins University Press.

Klein, Emilio y Víctor E. Tokman (1988), "Sector informal: una forma de utilizar el trabajo como consecuencia de la manera de producir y no viceversa. A propósito del artículo de Portes y Benton", Estudios Sociológicos, vol. 6, núm. 16, pp. 205-212.

Lápple, Dieter (1985), "Internationalization of Capital and the Regional Problem", en John Walton (ed.), Capital and Labour in the Urbanized World, Beverly Hills, SAGE Publications, pp. 43-75.

Lustig, Nora (1992), Mexico, The Remaking of an Economy, Washington D.C., The Brookings Institution.

Marshall, Adriana (1990), “¿Contracciones flexibles o trabajo precario? El empleo temporario y a tiempo parcial", en Pedro Galin y Marta Novick (comps.), La precarización del empleo en la Argentina, Buenos Aires, Centro Editor de América Latina, pp. 18-46.

- (1987), "Non-Standard Employment Practices in Latin America", International Institute for Labour Studies Discussion Papers, Ginebra, International Institute for Labour Studies.

Mezzera, Jaime (1987), "Crédito y capacitación para el sector informal", Investigaciones sobre Empleo, núm. 29, Santiago de Chile, PREALc.

Oliveira, Orlandina de (1989), "Empleo femenino en México en tiempos de recesión económica: tendencias recientes", Fuerza de trabajo femenina urbana en México, México, unAm/Porrúa, vol. 1, pp. 29-66.

Pérez Sảinz, Juan Pablo y Rafael Menjivar Larín (1991), "Informalidad urbana en Centroamérica: características estructurales y lógicas de funcionamiento", en Juan Pablo Pérez Sáinz y Rafael Menjívar Larín (comps.), Informalidad urbana en Centroamérica. Entre la acumulación y la subsistencia, San José de Costa Rica, Flacso/Editorial Nueva Sociedad, pp. 21-44.

Portes, Alejandro (1989), "La informalidad como parte integral de la economía moderna y no como indicador de atraso: respuesta Klein y Tokman", Estudios Sociológicos, vol. 7, núm. 20, pp. 369-374.

_ y Richard Schauffler (1993), "Competing Perspectives on the Latin American Informal Sector”, Population and Development Review, vol. 19, núm 1, pp. 33-60.

- y Michael Johns (1989), "The Polarization of Class and Space in the Contemporary Latin American City", en William L. Canak (ed.), Lost Promises. Debt, Austerity, and Development in Latin America, Boulder (Colorado), Westview Press, pp. 111-137.

PREALC (1991), Empleo y equidad: El desafio de los 90, Santiago de Chile, ort. 
(1987), El sector informal hoy: El imperativo de acluar, Santiago de Chile, ort. (1976), The Employment Problem in Latin America, Santiago de Chile, ort. Rendón, Teresa y Carlos Salas (1992), "El mercado de trabajo no agrícola en México. Tendencias y cambios recientes", en Ajuste estructural, mercados laborales y $T L C$, México, El Colegio de México/Fundacion Friedrich Ebert/El Colegio de la Frontera Norte, pp. 13-31.

___ y Carlos Salas (1987), "Evolución del empleo en México: 1895-1970", Estudios Demográficos y Urbanos, vol. 2, núm. 2, pp. 189-230.

Roberts, Bryan (1993), "Enterprise and Labor Markets: The Border and the Metropolitan Areas", Frontera Norte, vol. 5, núm. 9, pp. 33-65.

(1991), "The Changing Nature of Informal Employment: The Case of Mexico", en Guy Standing y Victor Tokman (eds.), Towards Social Adjustment. Labour Market Issues in Structural Adjustment, Ginebra, International Labour Office, pp. 115-140.

(1990), "The Informal Sector in Comparative Perspective", en M. Estellie Smith (ed.), Perspectives on the Informal Economy, Nueva York, University Press of America, pp. 23-48.

(1989), "The Other Working Class: Uncommitted Labor in Britain, Spain, and Mexico", en Melvin L. Kohn (ed.), Cross-National Research in Sociology, Beverly Hills, sAGE Publications, pp. 352-372.

Rodgers, Gerry (1989), "Introduction: Trends in Urban Poverty and Labour Market Access", en Gerry Rodgers (ed.), Urban Poverty and the Labour Market, Ginebra, International Labour Office, pp. 1-33.

Rosenberg, Samuel (1989), "Labor Market Restructuring in Europe and the United States: The Search for Flexibility", en Samuel Rosenberg (ed.), The State and the Labor Market, Nueva York-Londres, Plenum Press, pp. 3-16.

Telles, Edward (1988), The Consequences of Employment Structure Brazil: Earnings, Socio-Demographic Characteristics and Metropolitan Area Differences, Austin, University of Texas.

Tokman, Victor E. (1991), "The Informal Sector in Latin America: From Underground to Legality", en Guy Standing y Víctor Tokman (eds.), Towards Social Adjustment. Labour Market Issues in Structural Adjustment, Ginebra, International Labour Office, pp. 141-157.

(1987), "El sector informal: quince años después", El Trimestre Económico, vol. 54, núm. 3, pp. 513-536.

(1977), Dinámica del mercado de trabajo urbano: el sector informal urbano en América Latina, Santiago de Chile, olT/ PREAlc.

Walton, John (1985), "The Third New International Division of Labour", en John Walton (ed.), Capital and Labour in the Urbanized World, Beverly Hills, sace Publications, pp. 3-14.

Zenteno, René y Rodolfo Cruz (1992), "Boom in the Midst of the Crisis: the Strange Case of Weil-Being in Tijuana", trabajo presentado en el Seminario Sociodemographic Effects the 1980's Economic Crisis in Mexico, Austin, University of Texas (mimeo). 